\title{
$\mathrm{CTAB}$ 辅助水热合成球状纳米花 $\mathrm{MoS}_{2} / \mathrm{C}$ 复合材料 及其电化学性能研究
}

\author{
蔡亚菱, 李亚飞, 王增梅, 张耀, 陈坚, 郭新立 \\ (东南大学 材料科学与工程学院, 江苏省土木工程材料重点实验室, 南京 211189)
}

\begin{abstract}
摘 要: 以钿酸钠 $\left(\mathrm{Na}_{2} \mathrm{MoO}_{4} \cdot 2 \mathrm{H}_{2} \mathrm{O}\right)$ 、硫嫝 $\left(\mathrm{NH}_{2} \mathrm{CSNH}_{2}\right) 、 \mathrm{CTAB}$ 为原料, 利用水热法合成了 $\mathrm{MoS}_{2} / \mathrm{C}$ 球状纳米花复合 材料。通过 XRD、SEM、TEM、TG 等分析测试方法, 研究了不同 CTAB 添加量对 $\mathrm{MoS}_{2} / \mathrm{C}$ 复合材料的微观结构、 表面形貌的影响规律, 结果显示, 有部分无定形碳嵌入了 $\mathrm{MoS}_{2}$ 层间, 并抑制了 $\mathrm{MoS}_{2}(002)$ 面的堆积。电化学 测试表明: 与纯 $\mathrm{MoS}_{2}$ 相比, $\mathrm{MoS}_{2} / \mathrm{C}$ 复合材料具有更好的电化学性能, 当加入 $0.025 \mathrm{~g} \mathrm{CTAB}$ 时首次放电比容量达到 $730 \mathrm{mAh} / \mathrm{g}$, 在 $100 \mathrm{~mA} / \mathrm{g}$ 的电流密度下经过 100 次循环比容量稳定在 $415 \mathrm{mAh} / \mathrm{g}$ 。在此基础上讨论了 $\mathrm{MoS}_{2} / \mathrm{C}$ 球状 纳米花复合材料的可能生长机理以及对材料电化学性能的影响规律。
\end{abstract}

关 键 词: $\mathrm{MoS}_{2} / \mathrm{C}$ 复合材料; 锂离子电池; 负极材料

中图分类号: TQ174 文献标识码: A

\section{CTAB-assisted Synthesis of $\mathrm{MoS}_{2} / \mathrm{C}$ Nano-flowers with Improved Electrochemical Performances for Lithium Ion Batteries}

\author{
CAI Ya-Ling, LI Ya-Fei, WANG Zeng-Mei, ZHANG Yao, CHEN Jian, GUO Xin-Li \\ (Jiangsu Key Laboratory of Construction Materials, Southeast University, Nanjing 211189, China)
}

\begin{abstract}
MoS}_{2} / \mathrm{C}$ composites were prepared by a simple CTAB assistant thermal reduction method using sodium molybdate, thiourea and CTAB as starting materials. The samples were characterized by XRD, SEM, TEM, and TG. It is confirmed that the amorphous carbon is partially inserted into the interlayer spaces of molybdenum disulfide and inhibits the accumulation of (002) plane. Meanwhile, the electrochemical properties were studied in detail. As a consequence, the $\mathrm{MoS}_{2} / \mathrm{C}$ composites have better electrochemical performance than pure $\mathrm{MoS}_{2}$. The composite synthesized by adding $0.025 \mathrm{~g}$ CTAB shows the best electrochemical performance. The initial discharge capacity was $730 \mathrm{mAh} / \mathrm{g}$ and a stable capacity above $415 \mathrm{mAh} / \mathrm{g}$ was retained for 100 cycles tested at $100 \mathrm{~mA} / \mathrm{g}$. At last, the possible growth mechanism of the $\mathrm{MoS}_{2} / \mathrm{a}-\mathrm{C}$ composites and the influence of different micro-morphology are also calculated.
\end{abstract}

Key words: $\mathrm{MoS}_{2} / \mathrm{C}$ composites; lithium ion batteries; anode materials

锂离子电池是目前广泛使用的可充电移动电 源, 已被用于手机、笔记本电脑、数码相机等便携 式电子产品中。此外，它还在医学器件、电动汽车、 航空航天、国防军事等领域有着广泛的应用前景和 发展空间。但锂离子电池的负极材料仍存在很大的
改进空间, 其研究主要追求较低的嵌锂电位、较高 的比容量、优异的循环性能、较低的成本以及较高 的安全性。目前作为商业化锂离子石墨类负极材 料, 具有循环稳定性好、工作电压较高、成本低廉 等特点。然而石墨类负极材料的比容量较低, 仅为 
$372 \mathrm{mAh} / \mathrm{g}$, 不能满足高容量、持续大电流放电的能 力, 从而限制了锂离子电池的进一步发展 ${ }^{[1-2]}$ 。因此, 如何制备出性能更好的负极材料成了近年来锂离子 电池研究的热点。其中, $\mathrm{MoS}_{2}$ 具有与石墨相似的典 型层状结构: 层内 S-Mo-S 原子组成三明治型层状 结构, 层内由较强的共价键结合, 而层间则由较弱 的范德华力结合 ${ }^{[3]}$ 。正是由于 $\mathrm{MoS}_{2}$ 层间较弱的作用 力, 使得可以通过嵌入的方法在其层间引入外来分 子、原子或离子而不改变其层内结构。作为锂离子 电池负极材料, $\mathrm{MoS}_{2}$ 具有较高的比容量 ${ }^{[4]}$, 可达 669 1675 mAh/g, 其特殊的层状结构也利于 $\mathrm{Li}^{+}$快 速嵌入与脱出。然而, 由于 $\mathrm{MoS}_{2}$ 片层容易发生团聚 以及在 $\mathrm{Li}^{+}$嵌入和脱嵌的过程中会产生较大的体积 效应, 从而使电极结构不稳定, 导致电池容量衰减 快, 循环稳定性差 ${ }^{[5-6]}$ 。

为了提高 $\mathrm{MoS}_{2}$ 材料的导电性和循环稳定性, 一种常用的改性方法是制备 $\mathrm{MoS}_{2} / \mathrm{C}$ 复合材料。 Wang 等 ${ }^{[7]}$ 用水热法合成了 $\mathrm{MoS}_{2}$ 包覆碳纳米管的复 合材料, 在相同条件下, 50 次充放电循环后比容量 为 $400 \mathrm{mAh} / \mathrm{g}$, 大约是纯碳纳米管的 2 倍, 并展现了 良好的循环稳定性。Zhang 等 ${ }^{[8]}$ 以 $\mathrm{MoO}_{x} /$ 聚苯胺的混 合物为原料, 在 $\mathrm{H}_{2} \mathrm{~S}$ 气氛下热处理制备了一维 $\mathrm{MoS}_{2}-\mathrm{C}$ 纳米结构复合材料, 100 次充放电循环后比 容量保持在 $776 \mathrm{mAh} / \mathrm{g}$, 显示出优异的电化学性能。 Chang 等 ${ }^{[9]}$ 以葡萄糖为原料, 通过水热法合成了类 石墨烯状的 $\mathrm{MoS}_{2} / \mathrm{C}$ 复合材料, 100 次充放电循环后 比容量高达 $912 \mathrm{mAh} / \mathrm{g}$ 。显然, 不同的碳源类型对于 提高 $\mathrm{MoS}_{2} / \mathrm{C}$ 复合材料的电化学性能的影响是不同 的, 通过选择合适的碳源以及恰当的碳含量, 不但 可以提高 $\mathrm{MoS}_{2}$ 材料的比容量, 还可改善其循环稳 定性能。

本研究以钼酸钠、硫艮和不同添加量 CTAB 为 原料, 利用水热法合成了 $\mathrm{MoS}_{2} / \mathrm{C}$ 球状纳米花复合 材料, 然后在 $\mathrm{Ar}$ 和 $\mathrm{H}_{2}$ 的混合气氛下, $800^{\circ} \mathrm{C}$ 热处理 $4 \mathrm{~h}$ 后得到最终产物。并对其进行相关的结构形貌表 征和电化学性能分析测试。然后从不同 CTAB 添加 量的角度探讨了 $\mathrm{MoS}_{2} / \mathrm{C}$ 球状纳米花复合材料的可 能生长机理以及对材料电化学性能影响的规律。

\section{1 实验方法}

\section{1 $\mathrm{MoS}_{2} / \mathrm{C}$ 球状纳米花复合材料的制备}

将一定质量的 CTAB 加入到 $30 \mathrm{~mL}$ 去离子水中, 摚拌几分钟后, 将 $0.6 \mathrm{~g}$ 钼酸钠 $\left(\mathrm{Na}_{2} \mathrm{MoO}_{4} \cdot 2 \mathrm{H}_{2} \mathrm{O}\right)$ 和 $0.8 \mathrm{~g}$ 硫脲 $\left(\mathrm{NH}_{2} \mathrm{CSNH}_{2}\right)$ 加入到上述溶液中, 继续摚
拌至溶液澄清透明, 转移至 $100 \mathrm{~mL}$ 的聚四氟乙烯 和不锈钢制成的反应釜中密封, 在烘箱中 $200^{\circ} \mathrm{C}$ 下 加热 $24 \mathrm{~h}$ 。自然冷却到室温后过滤, 并用去离子水 和无水乙醇分别清洗样品, 然后将样品在 $60^{\circ} \mathrm{C}$ 下干 燥 $12 \mathrm{~h}$ 。最后将样品置于石英舟中, 在 $95 \%$ 氩气和 $5 \%$ 氢气混合气体的管式炉中 $800^{\circ} \mathrm{C}$ 下退火 $4 \mathrm{~h}$, 冷 却至室温取出。实验中, CTAB 的加入量分别为 $0.025 、 0.05 、 0.1 、 0.2$ 和 $0.3 \mathrm{~g}$ 。依次标记为 $\mathrm{MoS}_{2} / \mathrm{C}$ NF-0.025、 $\mathrm{MoS}_{2} / \mathrm{C}-\mathrm{NF}-0.05 、 \mathrm{MoS}_{2} / \mathrm{C}-\mathrm{NF}-0.1 、 \mathrm{MoS}_{2} /$ C-NF-0.2 和 $\mathrm{MoS}_{2} / \mathrm{C}-\mathrm{NF}-0.3$ 。同时作为对比, 在相同 条件下, 不加入 CTAB, 制备了纯 $\mathrm{MoS}_{2}$ 材料。

\section{$1.2 \mathrm{MoS}_{2} / \mathrm{C}$ 球状纳米花复合材料的表征}

采用 $\mathrm{X}$ 射线衍射仪(Bruker D8-Discover)分析 样品物相, 采用 SEM(FEI Sirion- 200 场发射扫描 电镜)和 TEM(FEI G2 20 透射电镜)分析结构形貌, 热重分析采用德国 STA 449 F3 型同步热分析仪, 空气气氛, 升温速率为 $10^{\circ} \mathrm{C} / \mathrm{min}$, 温度范围在室温 $1000^{\circ} \mathrm{C}$ 。

\section{$1.3 \mathrm{MoS}_{2} / \mathrm{C}$ 球状纳米花复合材料的电化学性} 能测试

电池的组装在充满氩气的手套箱中进行, 采用 电池壳型号为 2032 型扣式电池壳。将 $\mathrm{MoS}_{2} / \mathrm{C}$ 球状 纳米花状复合材料:乙炔黑:聚偏二氟乙烯 (PVDF)= 80wt\%:10wt\%:10wt\%混合均匀，然后 1-甲基-2-吡咯 烷酮(NMP)调成糊状均匀涂覆在铜簿上, 最后真空 干燥箱中 $80^{\circ} \mathrm{C}$ 干燥 $12 \mathrm{~h}$ 。以金属锂片作为对电极; 电解液为 $1 \mathrm{~mol} / \mathrm{L} \mathrm{LiPF}_{6} /(\mathrm{EC}+\mathrm{DMC})$ 的混合液, 其中 $\mathrm{EC}: \mathrm{DMC}=1: 1$ (体积比); 隔膜为 Celgard2400微孔聚 丙烯膜。

采用武汉蓝电 CT2001A 型电池测试系统进行 恒电流充放电测试, 电流密度为 $100 \mathrm{~mA} / \mathrm{g}$, 电压范 围为 $0.01 \sim 3 \mathrm{~V}$ 。

\section{2 结果与讨论}

\section{1 表面形貌与结构}

图 1 为由不同含量 $\mathrm{CTAB}$ 辅助水热法合成的 $\mathrm{MoS}_{2} / \mathrm{C}$ 复合材料的 XRD 图谱。从图中可以看出, 相 对于纯 $\mathrm{MoS}_{2}$ 材料, 样品在 $2 \theta=14.1^{\circ} 、 33.2^{\circ} 、 39.6^{\circ}$ 出现了明显的衍射峰, 分别对应于 $\mathrm{MoS}_{2}$ 的 (002)、 (100)、(103)面(JCPDS 37-1492)。经过热处理后其结 晶度将会得到很大提高。而加入 CTAB 后, 样品在 $2 \theta=28.7^{\circ}$ 处也出现衍射峰, 其对应于 $\mathrm{MoS}_{2}$ (004) 面。对应于 $2 \theta=14.1^{\circ}$ 的 $\mathrm{MoS}_{2}$ (002)面衍射峰的强度 随着加入 CTAB 添加量的增加而逐渐消失。这表明 
加入的 CTAB 会对 $\mathrm{MoS}_{2}(002)$ 面的堆积产生抑制作 用, 且加入量越多, 抑制作用越明显 ${ }^{[10]}$ 。而且随着 (002)面衍射峰强度的逐渐消失, 在 $2 \theta=8.9^{\circ}$ 及 $17.5^{\circ}$ 处开始出现了 2 个衍射峰, 分别标记为 $\mathrm{A}$ 和 $\mathrm{B}$, 根 据布拉格方程计算可知晶面间距 $d_{\mathrm{A}} \approx 0.99 \mathrm{~nm}, d_{\mathrm{B}} \approx$ $0.51 \mathrm{~nm}$, 而且 $d_{\mathrm{A}} \approx 2 d_{\mathrm{B}}$, 由于 $\mathrm{MoS}_{2}(002)$ 面间距约为 $0.62 \mathrm{~nm}$, 其值介于 $d_{\mathrm{A}}$ 和 $d_{\mathrm{B}}$ 之间, 这表明有碳元素 嵌入其层间距中, 而且由于实验中 CTAB 碳化温度 远低于石墨化的温度, 因此碳源将会以无定形碳的 形式存在 ${ }^{[11]}$, 而 $d_{\mathrm{A}}$ 应为嵌入碳源后 $\mathrm{MoS}_{2}(002)$ 面的 层间距, $d_{\mathrm{B}}$ 则为嵌入的碳源与 $\mathrm{MoS}_{2}$ 的层间距, 这种 推断可以在后面的 TEM 分析中得到验证。

如图 2 所示, 对 5 组样品进行了热重分析以确 定样品中的碳含量, 从热重曲线上可知样品存在 3 个失重过程, 对应的温度区间分别为 $50^{\circ} \mathrm{C}$ 左右、 $270^{\circ} \mathrm{C} \sim 500^{\circ} \mathrm{C}$ 和 $650^{\circ} \mathrm{C} \sim 1000^{\circ} \mathrm{C}$ 。其中, 在 $50^{\circ} \mathrm{C}$ 左右 的失重过程, 对应于吸附在样品中水分的蒸发; 在 $270{ }^{\circ} \mathrm{C} 500{ }^{\circ} \mathrm{C}$ 的失重过程, 是由于 $\mathrm{MoS}_{2}$ 和 $\mathrm{C}$ 在空 气中发生了氧化反应, 其中 $\mathrm{MoS}_{2}$ 发生的氧化反应 式如下所示 ${ }^{[12-13]}$ 。

$$
2 \mathrm{MoS}_{2}+7 \mathrm{O}_{2} \longrightarrow 2 \mathrm{MoO}_{3}+4 \mathrm{SO}_{2}
$$

而在 $650^{\circ} \mathrm{C} 1000^{\circ} \mathrm{C}$ 的失重过程, 对应于 $\mathrm{MoO}_{3}$ 的升华。通过 $\mathrm{MoO}_{3}$ 失重的量以及结合反应式(1), 计算出了 5 组样品中的碳含量, 结果如表 1 所示。 通过表 1 可以看出, 随着 CTAB 含量的增加, $\mathrm{MoS}_{2}$ 的含量在逐步减少, 而碳的含量逐渐增加。碳含量 增加, 将会对样品的循环性能产生两面性, 这一点将 在后面的电化学性能和形成机理分析有详细论述。

图 3 为不同样品的 SEM 照片, 可以看出所有样 品基本都呈现纳米花球块状颗粒形貌。而随着 CTAB 含量的增加, 可以明显看到颗粒发生团聚、

分散、再团聚的过程, 其粒径大小也经历由大到小, 再到大的过程。从纯 $\mathrm{MoS}_{2}$ 样品中, 可以看到明显的

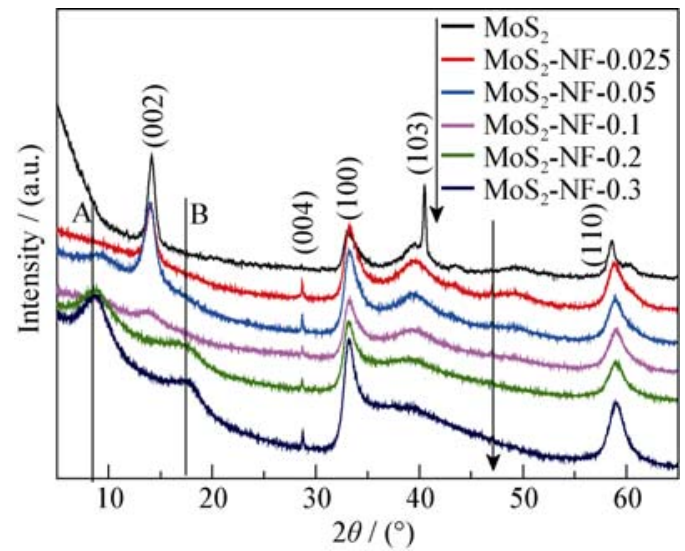

图 1 不同样品的 XRD 图谱

Fig. 1 XRD patterns of samples

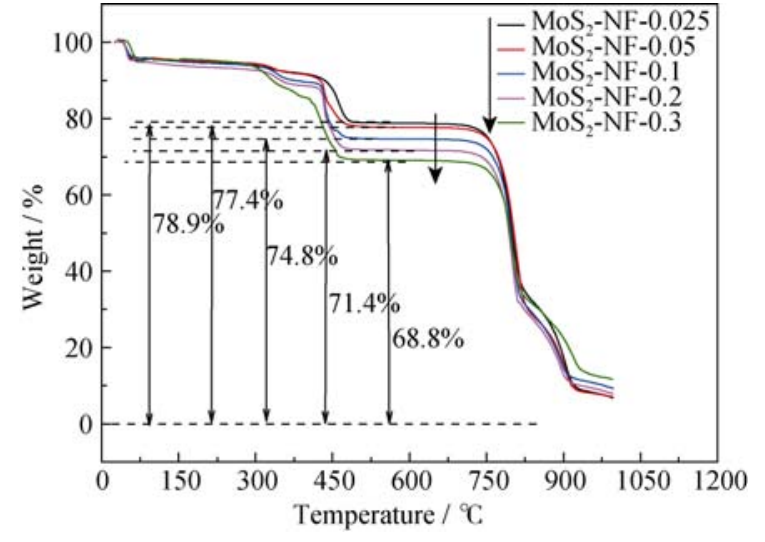

图 2 不同样品的热重曲线

Fig. 2 TGA curves of samples

表 1 样品中 $\mathrm{MoS}_{2}$ 和 $\mathrm{C}$ 含量

Table $1 \mathrm{MoS}_{2}$ and carbon content of samples

\begin{tabular}{cccc}
\hline Sample & $\mathrm{MoO}_{3} / \%$ & $\mathrm{MoS}_{2} / \%$ & $\mathrm{C} / \%$ \\
\hline $\mathrm{MoS}_{2} / \mathrm{C}-\mathrm{NF}-0.025$ & 78.9 & 87.6 & 12.4 \\
$\mathrm{MoS}_{2} / \mathrm{C}-\mathrm{NF}-0.05$ & 77.4 & 85.9 & 14.1 \\
$\mathrm{MoS}_{2} / \mathrm{C}-\mathrm{NF}-0.1$ & 74.8 & 83.0 & 17.0 \\
$\mathrm{MoS}_{2} / \mathrm{C}-\mathrm{NF}-0.2$ & 71.4 & 79.2 & 20.8 \\
$\mathrm{MoS}_{2} / \mathrm{C}-\mathrm{NF}-0.3$ & 68.8 & 76.3 & 23.7 \\
\hline
\end{tabular}
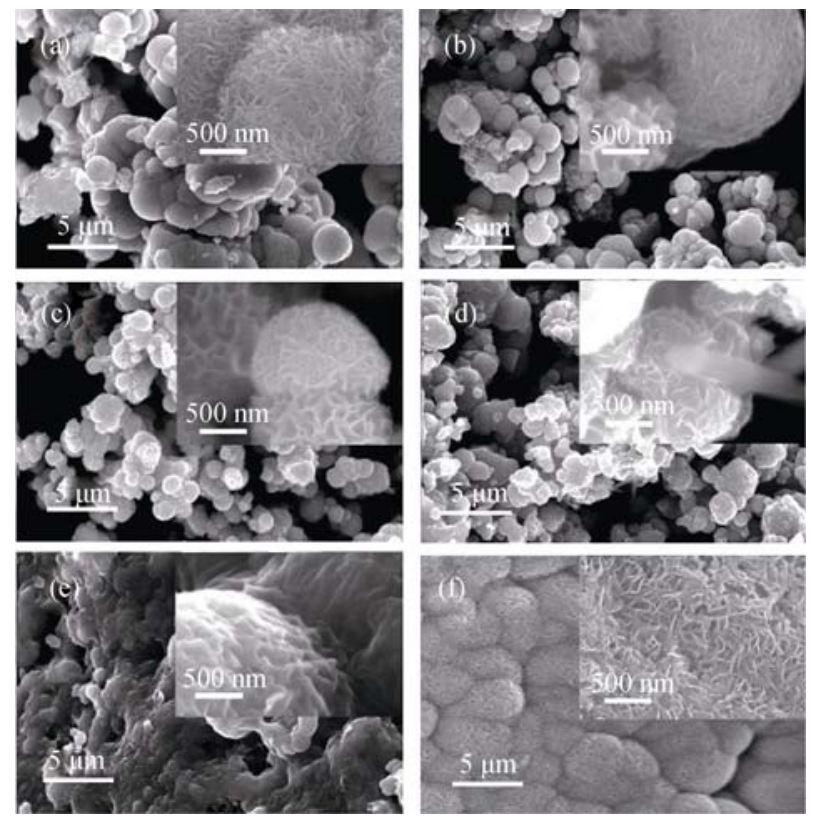

图 3 不同样品的 SEM 照片

Fig. 3 SEM images of samples

(a) $\mathrm{MoS}_{2} / \mathrm{C}-\mathrm{NF}-0.025$; (b) $\mathrm{MoS}_{2} / \mathrm{C}-\mathrm{NF}-0.05$; (c) $\mathrm{MoS}_{2} / \mathrm{C}-\mathrm{NF}-0.1$; (d) $\mathrm{MoS}_{2} / \mathrm{C}-\mathrm{NF}-0.2$; (e) $\mathrm{MoS}_{2} / \mathrm{C}-\mathrm{NF}-0.3$; (f) $\mathrm{MoS}_{2}$

$\mathrm{MoS}_{2}$ 片层结构。其中从 $\mathrm{MoS}_{2} / \mathrm{C}-\mathrm{NF}-0.025$ 样品可以 清晰地看到由薄而紧密的片层状 $\mathrm{MoS}_{2}$ 堆积而形成 纳米花状, 随着 CTAB 含量逐渐增加, 从 $\mathrm{MoS}_{2} / \mathrm{C}$ NF-0.05 和 $\mathrm{MoS}_{2} / \mathrm{C}-\mathrm{NF}-0.1$ 样品可以看出, 原本薄而 紧密的片层状 $\mathrm{MoS}_{2}$ 变得厚而疏松, 大而团聚的颗 
粒开始变得小而分散, 这种形貌的变化可能和碳源 的嵌入有关。一方面, 碳源的嵌入会使得 $\mathrm{MoS}_{2}$ 片层 变厚; 另一方面也会抑制 $\mathrm{MoS}_{2}$ 片层的堆积。从而使 得样品的形貌表现为厚而疏松且颗粒分散性较好。

而 $\mathrm{MoS}_{2} / \mathrm{C}-\mathrm{NF}-0.2$ 和 $\mathrm{MoS}_{2} / \mathrm{C}-\mathrm{NF}-0.3$ 样品几乎观察 不到片层状的纳米花球, 其形貌逐渐呈现球块状, 且团聚现象再次发生。这表明随着 CTAB 含量的进 一步增加, 其分散作用逐渐降低, 对样品的形貌有 着重要的影响。

图 4 为所有样品的 TEM 照片, 从样品中可以看 到明显的堆叠状片层结构。与纯 $\mathrm{MoS}_{2}$ 材料呈现紧 密堆积状不同, 在含有碳源的 $\mathrm{MoS}_{2}$ 样品中, 这种片 层堆积的更加疏松, 且分散性较好。而在呈片层状 的 $\mathrm{MoS}_{2}$ 区域中, 除了纯 $\mathrm{MoS}_{2}$ 材料外, 都可以看到 2 种层间距: $0.62 \mathrm{~nm}$ 和 $0.93 \mathrm{~nm}$, 其中 $0.62 \mathrm{~nm}$ 对应 为 $\mathrm{MoS}_{2}$ (002)面的层间距，而 $0.93 \mathrm{~nm}$ 的层间距则 与之前 XRD 图谱中 $\mathrm{A}$ 峰对应的层间距 $d_{\mathrm{A}}(\approx 0.99 \mathrm{~nm})$ 相当, 这充分表明了有部分无定形碳嵌入到 $M_{0} S_{2}$ 层间距中, 使得 $\mathrm{MoS}_{2}$ 层间距增大。与纯 $\mathrm{MoS}_{2}$ 材料 相比, 这种层间距增大可使比容量提高, 这一点可 以从随后电化学分析中看出。同时也可以观察到, 随着 CTAB 添加量的增加, $\mathrm{MoS}_{2}$ 片层堆积逐渐变得 稀疏, 分布也更加零散, $\mathrm{MoS}_{2}$ 层数的减少, 将会 使得 $\mathrm{Li}^{+}$可嵌入量减少, 从而降低了 $\mathrm{MoS}_{2}$ 容量。这 也说明了碳源的两面性: 一方面, 碳源的嵌入使得 $\mathrm{MoS}_{2}$ 层间距增大, 得以容纳更多的 $\mathrm{Li}^{+}$, 从而使得 容量提高; 另一方面, 碳源的存在也会抑制 $\mathrm{MoS}_{2}$ 片 层的堆积, 使得可嵌入的层数减少, 从而导致容量 降低, 而后面对不同含碳量 $\mathrm{MoS}_{2} / \mathrm{C}$ 复合材料的电 化学性能测试也可以印证这一点。此外, 随着含碳 量增加, 更多的碳源会嵌入 $\mathrm{MoS}_{2}$ 层间距中, 而堆积 层数将会进一步减少, 这也就解释了 XRD 图谱中 $\mathrm{MoS}_{2}(002)$ 面衍射峰的逐渐降低和消失, 而相应的 $\mathrm{A}$ 峰和 B 峰会逐渐增强的原因。

\section{2 生长机理分析}

基于以上分析测试结果, 本研究提出了 $\mathrm{CTAB}$ 辅助水热法制备 $\mathrm{MoS}_{2} / \mathrm{C}$ 球状纳米花的可能生长机 理(图 5)。在水热反应过程中主要发生了两个基本反 应：一是 CTAB 在水热条件下发生碳化，二是 $\mathrm{CS}\left(\mathrm{NH}_{2}\right)_{2}$ 与 $\mathrm{Na}_{2} \mathrm{MoO}_{4}$ 在水热条件下反应生成了 $\mathrm{MoS}_{2}$ 纳米片, 这两个反应同时进行。其中 CTAB 作 为一种常见的阳离子表面活性剂, 以合适的浓度溶 于水时会形成类球状胶束, 疏水基聚集在一起形成 内核，亲水基与水接触形成外壳 ${ }^{[14]}$ 。由于类球状的 胶束表面带正电, 通过静电作用吸附了溶液中的
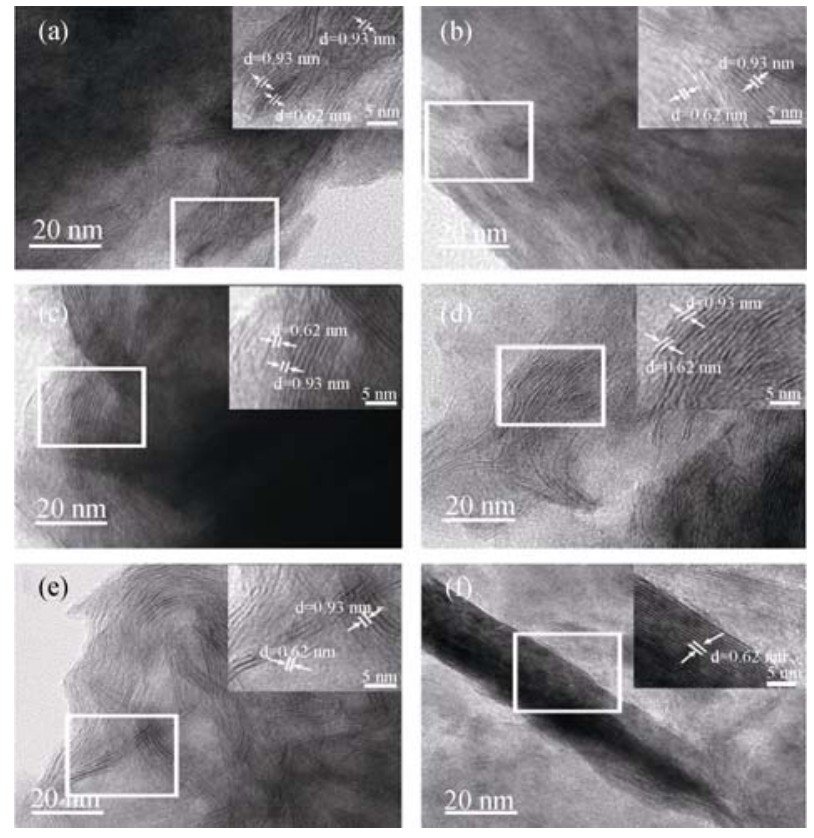

图 4 不同样品的 TEM 照片

Fig. 4 TEM images of samples (a) $\mathrm{MoS}_{2} / \mathrm{C}-\mathrm{NF}-0.025$; (b) $\mathrm{MoS}_{2} / \mathrm{C}-\mathrm{NF}-0.05$; (c) $\mathrm{MoS}_{2} / \mathrm{C}-\mathrm{NF}-0.1$; (d) $\mathrm{MoS}_{2} / \mathrm{C}-\mathrm{NF}-0.2$; (e) $\mathrm{MoS}_{2} / \mathrm{C}-\mathrm{NF}-0.3$; (f) $\mathrm{MoS}_{2}$

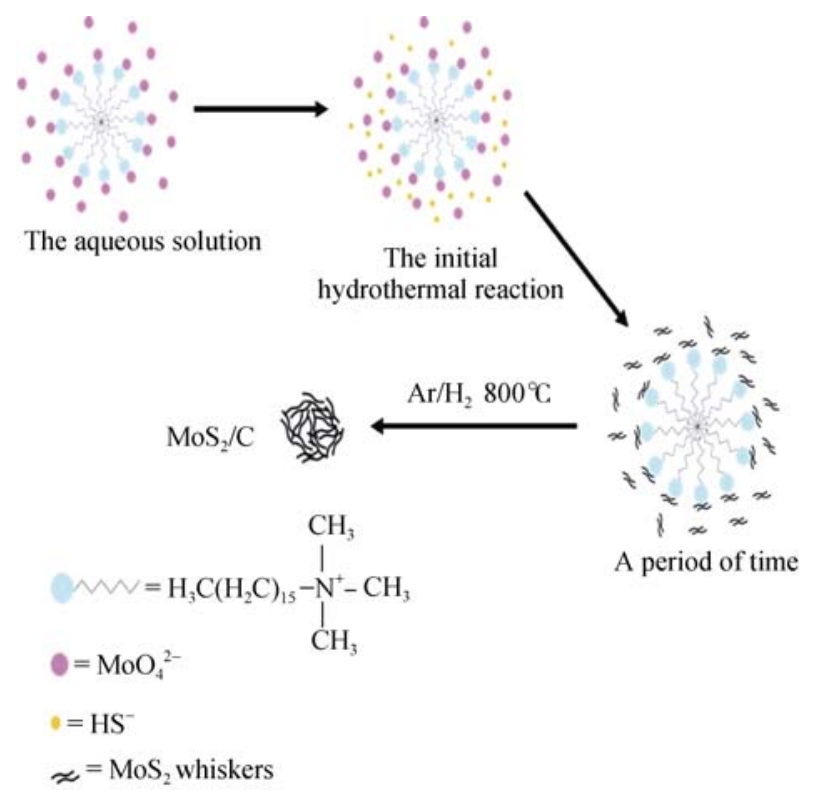

图 $5 \mathrm{MoS}_{2} / \mathrm{C}$ 球状纳米花的生长机理图

Fig. 5 Schematic illustration of the growth mechanisms of $\mathrm{MoS}_{2} / \mathrm{C}$ sample

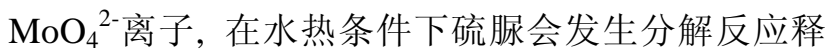
放 $\mathrm{H}_{2} \mathrm{~S}$ 气体, 与 $\mathrm{Na}_{2} \mathrm{MoO}_{4}$ 反应生成 $\mathrm{MoS}_{2}$ 纳米片, 总 的反应方程式如下所示 ${ }^{[15]}$ :

$$
\begin{gathered}
4 \mathrm{Na}_{2} \mathrm{MoO}_{4}+15 \mathrm{CS}\left(\mathrm{NH}_{2}\right)_{2}+6 \mathrm{H}_{2} \mathrm{O} \longrightarrow \\
4 \mathrm{MoS}_{2}+\mathrm{Na}_{2} \mathrm{SO}_{4}+6 \mathrm{NaSCN}+24 \mathrm{NH}_{3}+9 \mathrm{CO}_{2}
\end{gathered}
$$

然后 $\mathrm{MoS}_{2}$ 纳米片在类球状胶束表面形核、长 
大、堆垛, 在堆垛的过程中与 CTAB 碳化形成的碳 质材料交织在一起，在此过程中会有一部分碳源嵌 入其中，而这种相互交织作用也阻碍了水热反应过 程中 $\mathrm{MoS}_{2}$ 晶体结构的形成, 特别是(002)面的层状 结构的形成。而随着 CTAB 添加量的增加, 胶束的 分散性会降低而发生团聚现象, 使得最终形貌呈现 无规则的球块状且粒径较大。

\section{3 电化学分析测试}

图 6 为不同样品的前 3 次充放电曲线, 5 组样 品的充放电行为较为类似。以 $\mathrm{MoS}_{2} / \mathrm{C}-\mathrm{NF}-0.025$ 样 品为例, 阐释在其充放电过程中发生的反应。在首 次放电过程中, 样品在 $1.1 \mathrm{~V}$ 和 $0.6 \mathrm{~V}$ 处显示出 2 个 明显的电位平台。其中 $1.1 \mathrm{~V}$ 处的电位平台表明了 有 $\mathrm{Li}_{x} \mathrm{MoS}_{2}$ 生成, 对应的反应式如下 ${ }^{[4]}$ :

$$
\mathrm{MoS}_{2}+x \mathrm{Li}^{+}+x \mathrm{e}^{-} \longrightarrow \mathrm{Li}_{x} \mathrm{MoS}_{2}\left(\sim 1.1 \mathrm{~V} \text { vs. } \mathrm{Li} / \mathrm{Li}^{+}\right)
$$

而在 $0.6 \mathrm{~V}$ 处的电位平台则是 $\mathrm{Li}_{\mathrm{x}} \mathrm{MoS}_{2}$ 的原位 分解反应，其反应方程式如下:

$\mathrm{Li}_{x} \mathrm{MoS}_{2}+(4-x) \mathrm{e}^{-} \longrightarrow \mathrm{Mo}+2 \mathrm{Li}_{2} \mathrm{~S}\left(\sim 0.6 \mathrm{~V}\right.$ vs. $\left.\mathrm{Li} / \mathrm{Li}^{+}\right)$

在首次充电过程中, 样品在 $2.2 \mathrm{~V}$ 处有一个明 显的电位平台，其反应方程式如下:

$$
\mathrm{Li}_{2} \mathrm{~S} \longrightarrow 2 \mathrm{Li}^{+}+2 \mathrm{e}^{-}+\mathrm{S}\left(\sim 2.6 \mathrm{~V} \text { vs. } \mathrm{Li} / \mathrm{Li}^{+}\right)
$$

在经过首次充放电循环以后, $\mathrm{MoS}_{2}$ 完全转变为 $\mathrm{Mo}$ 和 $\mathrm{S}$ 单质。在随后的第 2 次和第 3 次放电曲线 中, 样品在 1.1 和 $0.6 \mathrm{~V}$ 处的放电平台消失, 而在
2.0、1.0 和 $0.4 \mathrm{~V}$ 处出现 3 个新的电位平台, 其中在 $2.0 \mathrm{~V}$ 处的放电平台对应 $\mathrm{S}$ 转变为 $\mathrm{Li}_{2} \mathrm{~S}$, 在 $1.1 \mathrm{~V}$ 处 的放电平台对应着 $\mathrm{Li}_{x} \mathrm{MoS}_{2}$ 的形成，在 $0.4 \mathrm{~V}$ 处的放 电电位则为 $\mathrm{Mo}^{4+}$ 进一步还原为 $\mathrm{Mo}$ 。同时可以发现 随着含碳量的增加, 样品的充放电曲线重合度逐渐 提高, 这也表明了制备的 $\mathrm{MoS}_{2} / \mathrm{C}$ 复合材料具有较 好的稳定性和作为锂离子电池负极材料的可行性。

图 7 为不同样品在 $100 \mathrm{~mA} / \mathrm{g}$ 电流密度下循环 100 次的容量曲线。从图中可以看出, 与纯 $\mathrm{MoS}_{2}$ 相 比, 通过水热法制备的 $\mathrm{MoS}_{2} / \mathrm{C}$ 复合材料具有更高 的比容量和更好的循环稳定性, 其中 $\mathrm{MoS}_{2} / \mathrm{C}-\mathrm{NF}-$ 0.025 样品拥有最高的首次放电比容量 $(975 \mathrm{mAh} / \mathrm{g})$, 而纯 $\mathrm{MoS}_{2}$ 的首次放电比容量仅为 $572 \mathrm{mAh} / \mathrm{g}$ 。虽然 $\mathrm{MoS}_{2} / \mathrm{C}-\mathrm{NF}-0.05$ 样品的首次放电比容量在所有 $\mathrm{MoS}_{2} / \mathrm{C}$ 复合材料中最低, 为 $730 \mathrm{mAh} / \mathrm{g}$, 但在 100 次 循环后, 循环稳定性最好, 比容量最高, 为 $415 \mathrm{mAh} / \mathrm{g}, \mathrm{MoS}_{2} / \mathrm{C}-\mathrm{NF}-0.3$ 样品最低, 比容量为 $250 \mathrm{mAh} / \mathrm{g}$, 而纯 $\mathrm{MoS}_{2}$ 经过 100 次循环后, 比容量仅 为 $35 \mathrm{mAh} / \mathrm{g}$, 这表明通过制备 $\mathrm{MoS}_{2} / \mathrm{C}$ 复合材料可以 有效地提高 $\mathrm{MoS}_{2}$ 材料的电化学性能。同时可以发现 随着含碳量的增加, $\mathrm{MoS}_{2} / \mathrm{C}$ 复合材料的容量稳定性 呈现先增加后减少的趋势, 其中 $\mathrm{MoS}_{2} / \mathrm{C}-\mathrm{NF}-0.05$ 材 料表现出最佳性能。这也印证了之前的分析，再次表 明碳源的作用具有两面性，合适的碳含量不但会增 加比容量，也会提高材料的循环稳定性。
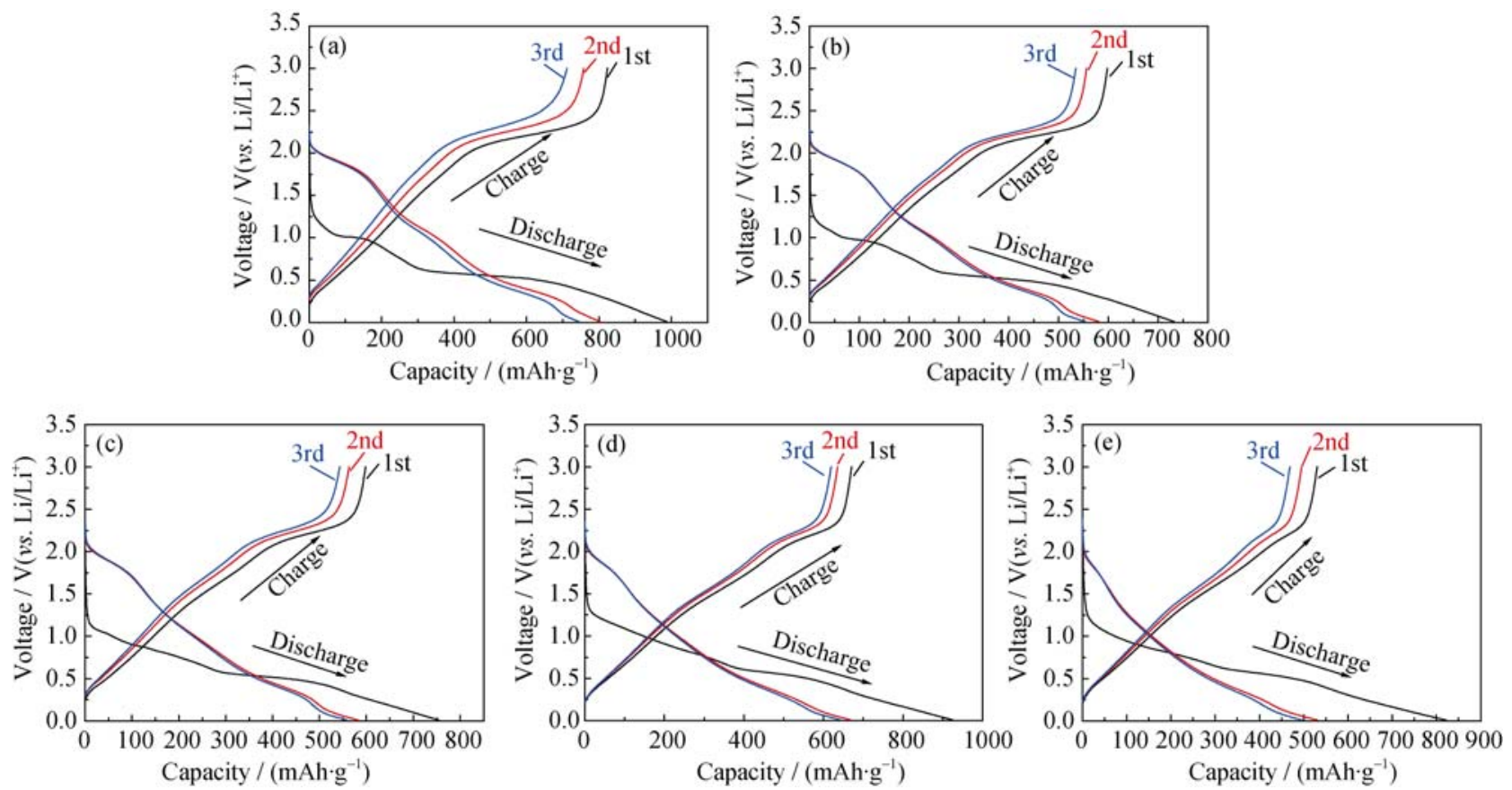

图 6 样品前 3 次的充放电曲线

Fig. 6 The initial three charge/discharge potential profiles of samples (a) $\mathrm{MoS}_{2} / \mathrm{C}-\mathrm{NF}-0.025$; (b) $\mathrm{MoS}_{2} / \mathrm{C}-\mathrm{NF}-0.05$; (c) $\mathrm{MoS}_{2} / \mathrm{C}-\mathrm{NF}-0.1$; (d) $\mathrm{MoS}_{2} / \mathrm{C}-\mathrm{NF}-0.2$; (e) $\mathrm{MoS}_{2} / \mathrm{C}-\mathrm{NF}-0.3$ 


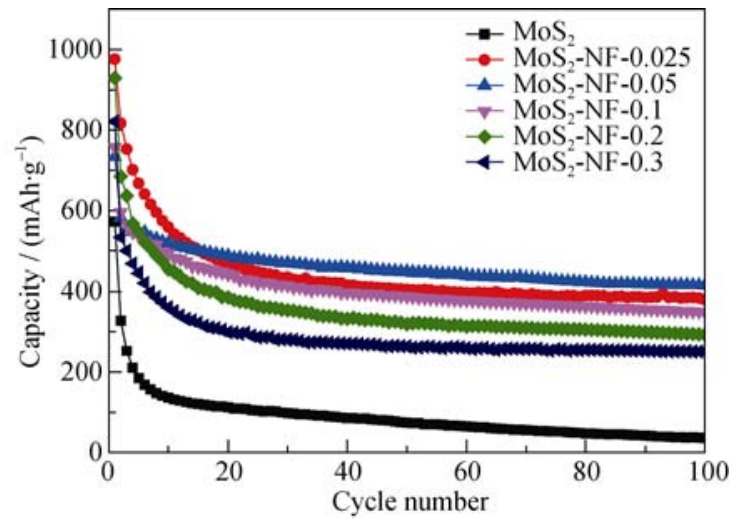

图 7 样品的循环特性曲线

Fig. 7 Cyclic performance of samples

\section{3 结论}

本研究利用 CTAB 辅助水热法并经热处理合成 了 $\mathrm{MoS}_{2} / \mathrm{C}$ 球状纳米花复合材料, 通过 XRD、SEM、 TEM、TG 分析测试表明, 随着碳含量的增加, 样品 的形貌有类球状变为球块状，粒径则经历了由大到 小再到大的变化过程。由于无定形碳的嵌入使得 $\mathrm{MoS}_{2}$ 层间增加，但也抑制了 $\mathrm{MoS}_{2}$ 片层(002)面片层 的堆积, 从而使得 $\mathrm{MoS}_{2}$ 纳米片层由薄而紧密逐渐 变为厚而稀疏。对样品的电化学性能测试表明, 与 纯 $\mathrm{MoS}_{2}$ 相比, $\mathrm{MoS}_{2} / \mathrm{C}$ 复合材料具有更高的比容量 和更好循环稳定性, 其中 $\mathrm{MoS}_{2} / \mathrm{C}-\mathrm{NF}-0.05$ 表现出最 好的电化学性能, 在 $100 \mathrm{~mA} / \mathrm{g}$ 的电流密度下, 首次 放电比容量达到 $730 \mathrm{mAh} / \mathrm{g}$, 循环 100 次后比容量 稳定在 $415 \mathrm{mAh} / \mathrm{g}$ 。这主要由于碳源含量合适，可以 充分与 $\mathrm{MoS}_{2}$ 反应，从而形成最佳的嵌层结构，有利 于缓解体积效应, 便于 $\mathrm{Li}^{+}$的嵌入与脱出, 从而表现 出最好的电化学性能。本研究表明, 利用水热法制 备的 $\mathrm{MoS}_{2} / \mathrm{C}-\mathrm{NF}-0.05$ 的复合材料是最理想的负极 材料。

\section{参考文献:}

[1] TARASCON J M, ARMAND M. Issues and challenges facing rechargeable lithium batteries. Nature, 2001, 414(6861): 359-367.

[2] GOODENOUGH J B, KIM Y. Challenges for rechargeable Li batteries. Chemistry of Materials, 2009, 22(3): 587-603.

[3] RAPOPORT L, FLEISCHER N, TENNE R. Applications of $\mathrm{WS}_{2}$
$\left(\mathrm{MoS}_{2}\right)$ inorganic nanotubes and fullerene-like nanoparticles for solid lubrication and for structural nanocomposites. Journal of Materials Chemistry, 2005, 15(18): 1782-1788.

[4] STEPHENSON T, LI Z, OLSEN B, et al. Lithium ion battery applications of molybdenum disulfide $\left(\mathrm{MoS}_{2}\right)$ nanocomposites. Energy \& Environmental Science, 2014, 7(1): 209-231.

[5] SEN U K, MITRA S. High-Rate and high-energy-density lithium-ion battery anode containing 2D $\mathrm{MoS}_{2}$ nanowall and cellulose binder. ACS Applied Materials \& Interfaces, 2013, 5(4): $1240-1247$.

[6] LYNDEN A. Self-assembled $\mathrm{MoS}_{2}$-carbon nanostructures: influence of nanostructuring and carbon on lithium battery performance. Journal of Materials Chemistry, 2012, 22(26): 12988-12992.

[7] WANG Q, LI J. Facilitated lithium storage in $\mathrm{MoS}_{2}$ overlayers supported on coaxial carbon nanotubes. The Journal of Physical Chemistry C, 2007, 111(4): 1675-1682.

[8] ZHANG C, WANG Z, GUO Z, et al. Synthesis of $\mathrm{MoS}_{2}-\mathrm{C}$ one-dimensional nanostructures with improved lithium storage properties. ACS applied materials \& interfaces, 2012, 4(7): 3765-3768.

[9] CHANG K, CHEN W, MA L, et al. Graphene-like $\mathrm{MoS}_{2}$ /amorphous carbon composites with high capacity and excellent stability as anode materials for lithium ion batteries. Journal of Materials Chemistry, 2011, 21(17): 6251-6257.

[10] LIANG K S, CHIANELLI R R, CHIEN F Z, et al. Structure of poorly crystalline $\mathrm{MoS}_{2}-\mathrm{A}$ modeling study. Journal of Non-crystalline Solids, 1986, 79(3): 251-273.

[11] 王 臻. 类石墨烯 $\mathrm{MoS}_{2}$ /石墨烯复合纳米材料的合成及其电化学 储锂性能的研究. 杭州: 浙江大学硕士学位论文, 2013.

[12] PARK S K, YU S H, WOO S, et al. A simple l-cysteine-assisted method for the growth of $\mathrm{MoS}_{2}$ nanosheets on carbon nanotubes for high-performance lithium ion batteries. Dalton Transactions, 2013, 42(7): 2399-2405.

[13] WANG Z, MA L, CHEN W, et al. Facile synthesis of $\mathrm{MoS}_{2} /$ graphene composites: effects of different cationic surfactants on microstructures and electrochemical properties of reversible lithium storage. RSC Advances, 2013, 3(44): 21675-21684.

[14] SUN X, LI Y. Colloidal carbon spheres and their core/shell structures with noble-metal nanoparticles. Angewandte Chemie International Edition, 2004, 43(5): 597-601.

[15] TIAN Y, HE Y, SHANG J, et al. Hydrothermal synthesis and characterization of laminar $\mathrm{MoS}_{2}$. Acta Chimica Sinica, 2004, 62(18): $1807-1810$ 